\title{
Article \\ Prospective Study on Incidence, Risk Factors and Outcome of Recurrent Clostridioides difficile Infections
}

\author{
Guido Granata ${ }^{1}{ }^{4}$, Nicola Petrosillo ${ }^{1, *} \mathbb{0}$, Lucia Adamoli ${ }^{2}$, Michele Bartoletti ${ }^{3}$, Alessandro Bartoloni ${ }^{4}{ }^{(}$,

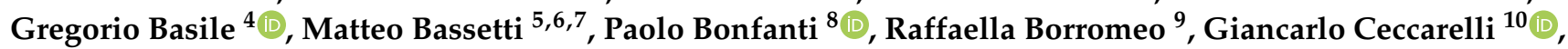

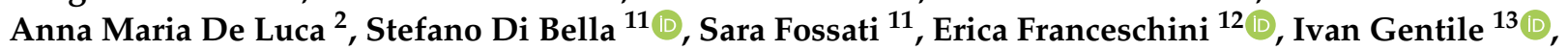 \\ Daniele Roberto Giacobbe ${ }^{5,6}{ }^{\infty}$, Enrica Giacometti ${ }^{7}$, Fabrizio Ingrassia ${ }^{9}$, Filippo Lagi ${ }^{4}$, Giambattista Lobreglio ${ }^{14}$, \\ Andrea Lombardi ${ }^{15}\left(\mathbb{D}\right.$, Laura Isabella Lupo ${ }^{14}\left(\mathbb{D}\right.$, Roberto Luzzati ${ }^{11}\left(\mathbb{D}\right.$, Alberto Enrico Maraolo ${ }^{13}(\mathbb{D}$, \\ Malgorzata Mikulska ${ }^{5,6}$, Mario Umberto Mondelli ${ }^{15}$, Alessandra Mularoni ${ }^{2}$, Cristina Mussini ${ }^{12}$, \\ Alessandra Oliva ${ }^{10}{ }^{\circledR}$, Alessandro Pandolfo ${ }^{16}$, Carlotta Rogati ${ }^{12}$, Filippo Fabio Trapani ${ }^{3}$, Mario Venditti ${ }^{10}$, \\ Pierluigi Viale ${ }^{3}$, Emanuela Caraffa ${ }^{1} \mathbb{D}$, Maria Adriana Cataldo ${ }^{1}$ and on behalf of the ReCloDi (Recurrence of \\ Clostridioides difficile Infection) Study Group ${ }^{+}$
}

\section{check for} updates

Citation: Granata, G.; Petrosillo, N.; Adamoli, L.; Bartoletti, M.; Bartoloni, A.; Basile, G.; Bassetti, M.; Bonfanti, P.; Borromeo, R.; Ceccarelli, G.; et al. Prospective Study on Incidence, Risk Factors and Outcome of Recurrent Clostridioides difficile Infections. J. Clin. Med. 2021, 10, 1127. https://doi.org/ $10.3390 / \mathrm{jcm} 10051127$

Academic Editors: Benoit Guery and Michela Sabbatucci

Received: 29 December 2020

Accepted: 19 February 2021

Published: 8 March 2021

Publisher's Note: MDPI stays neutral with regard to jurisdictional claims in published maps and institutional affiliations.

Copyright: (c) 2021 by the authors Licensee MDPI, Basel, Switzerland. This article is an open access article distributed under the terms and conditions of the Creative Commons Attribution (CC BY) license (https:// creativecommons.org/licenses/by/ $4.0 /)$
1 Clinical and Research Department for Infectious Diseases, National Institute for Infectious Diseases L. Spallanzani IRCCS, 00149 Rome, Italy; guido.granata@inmi.it (G.G.); emanuela.caraffa@inmi.it (E.C.); adriana.cataldo@inmi.it (M.A.C.)

2 Infectious Diseases ISMETT IRCCS, 90127 Palermo, Italy; ladamoli@ismett.edu (L.A.); lucia.adamoli5@gmail.com (A.M.D.L.); amularoni@ismett.edu (A.M.)

3 Department of Medical and Surgical Sciences, "Alma Mater Studiorum”, IRCCS S. Orsola Teaching Hospital, University of Bologna, 40126 Bologna, Italy; bartolettimichele@yahoo.it (M.B.); f.f.trapani@gmail.com (F.F.T.); pierluigi.viale@unibo.it (P.V.)

4 Department of Experimental and Clinical Medicine, University of Florence, 50121 Florence, Italy; alessandro.bartoloni@unifi.it (A.B.); sciglianobasile@hotmail.it (G.B.); filippo.lagi@gmail.com (F.L.)

5 Department of Health Sciences (DISSAL), University of Genoa, 16126 Genoa, Italy; matteo.bassetti@unige.it (M.B.); daniele.roberto.giacobbe@gmail.com (D.R.G.); m.mikulska@unige.it (M.M.) Infectious Diseases Unit, San Martino Polyclinic Hospital-IRCCS, 16132 Genoa, Italy

7 Infectious Diseases Clinic, Department of Medicine University of Udine and Azienda Sanitaria Universitaria Integrata di Udine, 33100 Udine, Italy; enrica.giacometti@gmail.com

8 Department of Infectious Diseases, San Gerardo Hospital, Monza-University of Milano-Bicocca, 20126 Milan, Italy; bonfanti.paolo@gmail.com

9 Hospital of Crema, 26013 Crema, Italy; raffaellaborromeo@gmail.com (R.B.); fabrizio.ingrassia@asst-crema.it (F.I.)

10 Department of Public Health and Infectious Diseases, Sapienza University of Rome, Policlinico Umberto I, 00185 Rome, Italy; giancarlo.ceccarelli@uniroma1.it (G.C.); alessandra.oliva@uniroma1.it (A.O.); mario.venditti@uniroma1.it (M.V.)

11 Infectious Diseases Department, Azienda Sanitaria Universitaria Integrata di Trieste, 34128 Trieste, Italy; stefano932@gmail.com (S.D.B.); fossatisara@gmail.com (S.F.); roberto.luzzati@asuits.sanita.fvg.it (R.L.)

12 Clinic of Infectious Diseases, University of Modena and Reggio Emilia, 41121 Modena, Italy; ericafranceschini0901@gmail.com (E.F.); cristina.mussini@unimore.it (C.M.); carlotta.rogati@gmail.com (C.R.)

13 Section of Infectious Diseases, Department of Clinical Medicine and Surgery, University of Naples "Federico II", 80138 Naples, Italy; ivan.gentile@unina.it (I.G.); albertomaraolo@mail.com (A.E.M.)

14 Vito Fazzi Hospital, 73100 Lecce, Italy; patologiaclinica.polecce@ausl.le.it (G.L.); lauraisalupo@gmail.com (L.I.L.)

15 Division of Infectious Diseases and Immunology, Fondazione IRCCS Policlinico San Matteo, 27100 Pavia, Italy; andrea.lombardi02@universitadipavia.it (A.L.); M.Mondelli@smatteo.pv.it (M.U.M.)

16 Infectious Diseases, ASST Lecco Hospital, 23900 Lecco, Italy; a.pandolfo@asst-lecco.it

* Correspondence: nicola.petrosillo@inmi.it; Tel.: +39-0655-170-432

$\dagger$ Membership of the ReCloDi (Recurrence of Clostridioides difficile Infection) Study Group is provided in the Acknowledgments.

Abstract: Background: Limited and wide-ranging data are available on the recurrent Clostridioides difficile infection (rCDI) incidence rate. Methods: We performed a cohort study with the aim to assess the incidence of and risk factors for rCDI. Adult patients with a first CDI, hospitalized in 15 Italian hospitals, were prospectively included and followed-up for $30 \mathrm{~d}$ after the end of antimicrobial treatment for their first CDI. A case-control study was performed to identify risk factors associated 
with 30-day onset rCDI. Results: Three hundred nine patients with a first CDI were included in the study; 32\% of the CDI episodes (99/309) were severe/complicated; complete follow-up was available for 288 patients (19 died during the first CDI episode, and 2 were lost during follow-up). At the end of the study, the crude all-cause mortality rate was $10.7 \%$ (33 deaths $/ 309$ patients). Two hundred seventy-one patients completed the follow-up; rCDI occurred in $21 \%$ of patients $(56 / 271)$ with an incidence rate of 72/10,000 patient-days. Logistic regression analysis identified exposure to cephalosporin as an independent risk factor associated with rCDI (RR: 1.7; 95\% CI: 1.1-2.7, $p=0.03$ ). Conclusion: Our study confirms the relevance of rCDI in terms of morbidity and mortality and provides a reliable estimation of its incidence.

Keywords: Clostridioides difficile; recurrence; risk factors; outcome; incidence

\section{Introduction}

The Gram-positive anaerobic bacterium Clostridioides difficile (CD) is a leading cause of nosocomial diarrhea worldwide, resulting in significant morbidity, mortality and prolonged hospital stay [1-3]. Recurrence of Clostridioides difficile infection (rCDI) is associated with a higher risk of death and higher hospitalization costs [1,2,4-6]; subsequent rCDI episodes represent a real "spiral of disease", and studies assessing the quality of life of patients with rCDI show that these patients live in constant concern of developing subsequent rCDI [7].

Clinical studies show wide-ranging rCDI rates after the primary CDI of $12 \%$ to $40 \%$ [8-10]. There is also an increased risk following any further recurrence of up to $64 \%$ [8-12]. However, scanty data are available on rCDI rates in Italy.

New, innovative treatment approaches, either antimicrobial or non-antimicrobial (e.g., monoclonal anti-toxin antibodies, microbiota transplantation, therapies with living bacteria and vaccines for $C D$ ), are in development. In the near future, these new therapies will represent effective alternatives in fighting and preventing rCDI. Therefore, efforts are needed to collect information on the burden of recurrence in wide populations in different countries and settings.

We performed a prospective multicenter cohort study with the objectives of assessing the 30-day onset rCDI rate in Italy, describing rCDI characteristics and outcomes and assessing the risk factors associated with rCDI.

\section{Materials and Methods}

\subsection{Study Site, Design, Population and Variables}

A prospective cohort study was performed in 15 Italian hospitals, including academic or tertiary referral hospitals. A complete list of participating centers is available as supplementary material (Supplementary Table S1), and their geographical distribution is shown in Figure 1.

All adult patients (age $>18$ y) admitted to the participating centers from January 2018 to March 2020 with a diagnosis of a first CDI episode were included in the study [13]. All included patients were followed-up for $30 \mathrm{~d}$ after the end of antimicrobial treatment for their first CDI episode (detailed in Figure 2).

For each enrolled patient, the following data were prospectively collected at study inclusion: age and gender; date of hospital admission; date of diarrhea onset and CDI diagnosis; hospitalization in the 3 months before the CDI diagnosis; treatment with antibiotics, antiacids, statins and steroids in the 3 months prior to CDI diagnosis; Charlson Comorbidity Index (CCI) at baseline and presence of co-morbidities.

The following laboratory findings were registered at the time of CDI diagnosis: serum creatinine, serum albumin levels, white blood cell count and peripheral neutrophil count.

Data were also collected on the severity, treatment and outcome of the first CDI episode.

During the 30-day follow-up, trained healthcare personnel assessed patients for the occurrence of rCDI; data on antibiotic exposure were also collected. In the case of hospital 
discharge before the end of follow-up, patients were contacted by phone call. Laboratory findings and clinical data of patients reporting rCDI diagnosis after hospital discharge were evaluated by the study investigators at the participating center, who decided whether or not to confirm the rCDI diagnosis according to the criteria reported below.

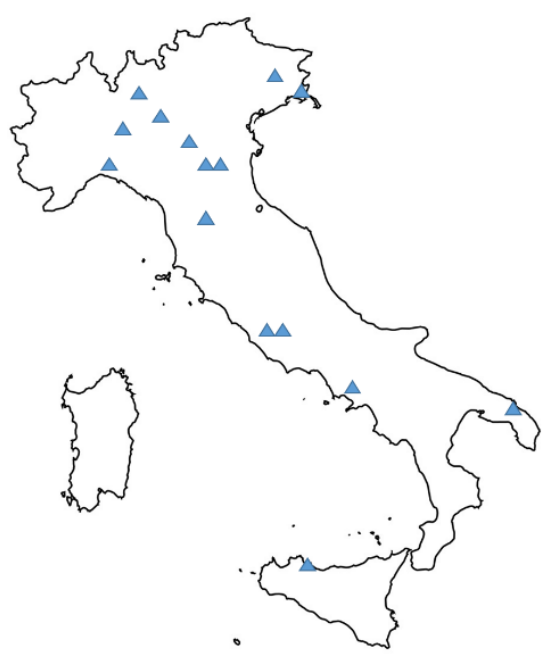

Figure 1. Geographical distribution of participating centers. A detailed list of the 15 participating centers is available as Supplementary Material (Table S1).

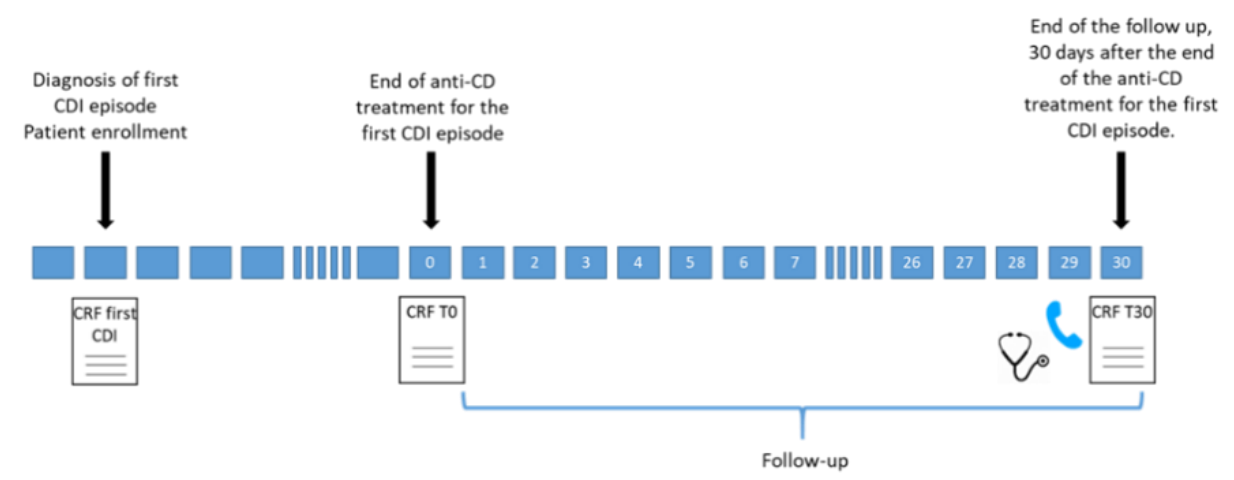

Figure 2. Details of the study follow-up. CDI: Clostridioides difficile infection. CRF: case report form.

When a diagnosis of rCDI was confirmed during the 30-day follow-up period, data were collected on the severity and treatment of the rCDI episode. Available laboratory findings were also collected, including serum creatinine levels, serum albumin levels, white blood cell count and peripheral neutrophil count, at the time of onset of rCDI. Finally, mortality at the end of follow-up was recorded.

To assess potential risk factors associated with rCDI, patients with rCDI were compared with patients who completed the follow-up and who did not present a recurrence of $\mathrm{CDI}$ in the 30-day period after the end of primary anti-CDI treatment.

\subsection{Definitions of CDI, Severe CDI and rCDI}

CDI was diagnosed by considering both microbiological results and clinical information: (1) the presence of diarrhea or evidence of megacolon or severe ileus and (2) a positive laboratory diagnostic test result (e.g., toxin enzyme immunoassay (EIA) or nucleic acid amplification test (NAAT)).

Severe CDI was defined as an episode of CDI with the presence of at least one of the following criteria: fever $\left(>38.5^{\circ} \mathrm{C}\right)$, chills, hemodynamic instability, signs of ileus or peritonitis, leukocytosis (leukocytes $>15,000$ cells $/ \mu \mathrm{L}$ ), increase in creatininemia $>1.5$ times the value 
before infection onset, increase in serum lactates, evidence of pseudo-membranous colitis and radiological incidence of ileus or ascites [13].

In our study, CDI recurrence was considered when CDI re-occurred within $30 \mathrm{~d}$ after the end of treatment for the first CDI episode, provided symptoms from the previous episode resolved after completion of initial treatment.

\subsection{Statistical Analysis}

Quantitative variables were tested for normal distribution and compared by means of a two-tailed test. Differences in groups were assessed using a $\chi^{2}$ test and Fisher's exact test. Precision of the risk ratio (RR) was determined by calculating a $95 \%$ confidence interval $(95 \% \mathrm{CI})$. A p-value of less than 0.05 was considered statistically significant. Variables from univariate analyses were considered for inclusion in multivariate logistic regression analysis if p-values were less than 0.05 . Backward stepwise logistic regression was performed, and the model that was considered biologically plausible and had the lowest -2 log-likelihood ratio was chosen as the final model.

Statistical analysis was performed using the software program Inter-cooled Stata (Stata Statistical Software, version 15).

\subsection{Ethical Considerations}

The study was first approved by the Ethics Committee of the coordinating center (National Institute for Infectious Diseases "L. Spallanzani", IRCCS, Rome; Ethics Committee registry number 543 23/01/2018) and, subsequently, by the Ethics Committees of the other 14 participating centers. Informed consent was obtained from each enrolled patient.

\section{Results}

Over the study period, 314 adult patients with a first CDI episode were enrolled; 309 patients gave informed consent and were included in the study. Two hundred eightyeight patients were assessed at the end of antimicrobial treatment for their first CDI episode, and 271 patients completed the 30-day follow-up for a total of 7795 patient-days (Figure 3).

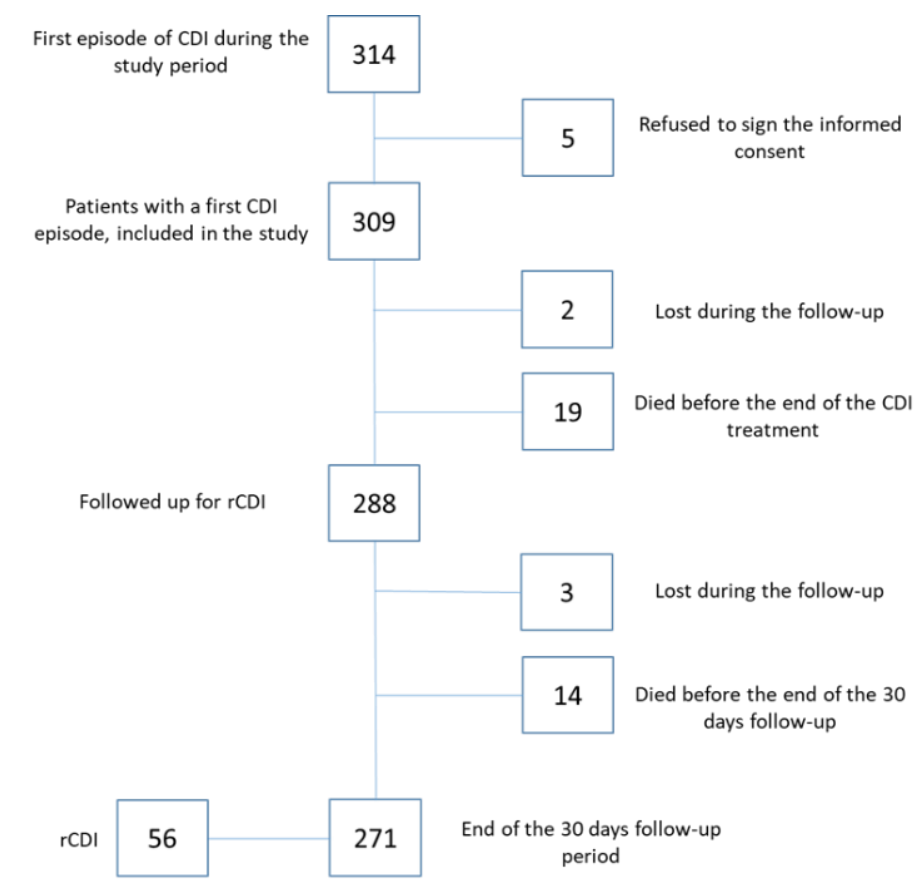

Figure 3. Follow-up of patients included in the study. CDI: Clostridioides difficile infection; rCDI: recurrent Clostridioides difficile infection. 


\subsection{Patients with a First CDI Episode}

Table 1 shows the characteristics of 309 patients with a first CDI episode. Thirty-two percent of them (99/309) were severe/complicated.

Table 1. Baseline characteristics of the 309 patients with a first CDI included in the study. CCI: Charlson Comorbidity Index; SD: standard deviation; CDI: Clostridioides difficile infection; COPD: chronic obstructive pulmonary disease; LTCF: long-term care facility. SD: standard deviation.

\begin{tabular}{|c|c|}
\hline Variables & CDI Patients (\%) \\
\hline Female gender & $152(46.2 \%)$ \\
\hline Age $(y)$ & 70 (range: $18-95$ ) \\
\hline Hospital onset of CDI & $168(54.4 \%)$ \\
\hline Comorbidities & $286(92.6 \%)$ \\
\hline - Cardiovascular disease & $168(54.3 \%)$ \\
\hline - Heart failure & $55(17.8 \%)$ \\
\hline - Diabetes & $75(24.2 \%)$ \\
\hline - $\quad$ Renal failure & $57(18.4 \%)$ \\
\hline - Dialysis & $12(3.98 \%)$ \\
\hline - Chronic liver failure & $30(9.1 \%)$ \\
\hline - $\quad$ Neurological disease & $52(16.8 \%)$ \\
\hline - $\quad$ Vasculitis & $9(2.9 \%)$ \\
\hline - $\quad \mathrm{COPD}$ & $69(22.3 \%)$ \\
\hline - $\quad$ Solid cancer & $42(13.6 \%)$ \\
\hline - Hematologic cancer & $23(7.4 \%)$ \\
\hline - Transplant, immunodeficiency, immunosuppression & $39(12.6 \%)$ \\
\hline - Other concomitant infections & $103(33.3 \%)$ \\
\hline Mean age-adjusted CCI & 5.3 (range: $0-13$ ) \\
\hline Hospitalization in the previous 3 months & $164(53 \%)$ \\
\hline Transferred to the hospital from a LTCF & $34(11 \%)$ \\
\hline Antibiotic administration in the previous 3 months & $220(71.2 \%)$ \\
\hline Antiacids administration in the previous 3 months & $214(69.2 \%)$ \\
\hline Statins administration in the previous 3 months & $77(24.9 \%)$ \\
\hline Steroids administration in the previous 3 months & $85(27.5 \%)$ \\
\hline Mean baseline serum creatinine $(\mathrm{mg} / \mathrm{dL} \pm \mathrm{SD})$ & $1.4 \pm 1.4$ \\
\hline Mean baseline serum albumin $(\mathrm{g} / \mathrm{dL} \pm \mathrm{SD})$ & $2.9 \pm 0.7$ \\
\hline Antibiotic administration at CDI diagnosis & $191(61.8 \%)$ \\
\hline \multicolumn{2}{|l|}{ CDI severity at diagnosis } \\
\hline - $\quad$ Mild CDI & $210(68.0 \%)$ \\
\hline - Severe/complicated CDI & $99(32.0 \%)$ \\
\hline \multicolumn{2}{|l|}{ Mean laboratory findings at CDI diagnosis } \\
\hline - $\quad$ Total peripheral white blood cell count $\left(10^{3}\right.$ cells $\left./ \mu \mathrm{L} \pm \mathrm{SD}\right)$ & $12.03 \pm 9.40$ \\
\hline - $\quad$ Peripheral neutrophil count $\left(10^{3}\right.$ cells $\left./ \mu \mathrm{L} \pm \mathrm{SD}\right)$ & $9.64 \pm 7.85$ \\
\hline - $\quad$ Serum creatinine $(\mathrm{mg} / \mathrm{dL} \pm \mathrm{SD})$ & $1.4 \pm 1.4$ \\
\hline - $\quad$ Serum albumin $(\mathrm{g} / \mathrm{dL} \pm \mathrm{SD})$ & $2.9 \pm 0.7$ \\
\hline
\end{tabular}


Regarding patient outcomes, at the end of antimicrobial treatment for the first CDI episode, 153/309 (49.5\%) patients were discharged home, 102/309 (33\%) were still in the hospital and 35/309 (11.3\%) were transferred to a long-term health-care facility, while $19 / 309(6.1 \%)$ patients died during the CDI episode (Table 1$)$.

Characteristics of the $288 \mathrm{CDI}$ patients at the end of the anti-CDI treatment are shown in Table 2. For the first CDI episode, the most common anti-CDI antimicrobial was vancomycin $(202 / 288,70.1 \%$ of cases), followed by metronidazole $(36 / 288,12.5 \%)$ and a combination of vancomycin and metronidazole (33/288, 11.5\%) (Table 2). At the end of antimicrobial treatment for the first CDI episode, 3/288 (1\%) and 4/288 (1.4\%) CDI patients needed surgery for complicated CDI and intensive care admission, respectively (Table 2).

Table 2. Characteristics of the 288 patients with a first CDI at the end of anti-CDI treatment for their first CDI episode and resolution of diarrhea. SD: standard deviation. PO: per os. IV: intravenous administration.

\begin{tabular}{|c|c|}
\hline Variables & Number of CDI Patients (\%) \\
\hline \multicolumn{2}{|l|}{ Administered anti-CD antimicrobial treatment } \\
\hline - Vancomycin & $202(70.1 \%)$ \\
\hline - $\quad$ PO Metronidazole & $36(12.5 \%)$ \\
\hline - $\quad$ Vancomycin and IV metronidazole & $33(11.5 \%)$ \\
\hline - $\quad$ Vancomycin and IV metronidazole and fidaxomicin & $5(1.7 \%)$ \\
\hline - Vancomycin and fidaxomicin & $7(2.4 \%)$ \\
\hline - $\quad$ Vancomycin and tigecycline & $2(0.6 \%)$ \\
\hline - $\quad$ Fidaxomicin & $1(0.3 \%)$ \\
\hline - Vancomycin and IV metronidazole and tigecycline & $2(0.6 \%)$ \\
\hline Albumin administration & $35(12.1 \%)$ \\
\hline Surgery for complicated CDI & $3(1 \%)$ \\
\hline Necessity of intensive care & $4(1.4 \%)$ \\
\hline Mean length of hospital stay of the discharged patients (d) & 23 (range: $1-167$ ) \\
\hline
\end{tabular}

\subsection{Patients with $r C D I$}

Two hundred seventy-one patients completed the 30-day follow-up. During this period, a rCDI episode occurred in 56 of them (21\%), with an incidence rate of 71.8 episodes per 10,000 patient-days.

Table 3 shows the characteristics of the 56 rCDI patients.

Their mean age was 70 y (range 29-85); 89\% of them had one or more co-morbidities (50/56). In 22 of them (39\%), their first CDI episode was severe or complicated.

During the follow-up period, 22/56 rCDI patients (39.2\%) received antibiotics. Only in $14(63.6 \%)$ of these was it possible to assess indication for antibiotic treatment, including pneumonia, abdominal infection, tuberculosis, osteomyelitis and urinary tract infection in $5(22.7 \%), 4(18.2 \%), 3(13.6 \%), 1(4.5 \%)$ and $1(4.5 \%)$ of the patients, respectively.

Thirteen (23.2\%) rCDI episodes were defined as severe/complicated. Treatment comprised vancomycin, fidaxomicin, a combination of vancomycin plus metronidazole and metronidazole in $34(60.7 \%), 10(17.8 \%), 7(12.5 \%)$ and $2(3.5 \%)$ cases, respectively. Seven out of $56(12.5 \%)$ rCDI cases also received albumin during anti-rCDI treatment (Table 3).

Regarding the rCDI outcome, 50 out of 56 (89.3\%) rCDI cases recovered. Six out of 56 (10.7\%) patients died. 
Table 3. Outcomes and characteristics of the 56 rCDI patients. SD: standard deviation. rCDI: recurrent Clostridioides difficile infection. PO: per os. IV: intravenous administration.

\begin{tabular}{|c|c|}
\hline Variables & $\begin{array}{l}\text { Number of rCDI } \\
\text { Patients }(\%)\end{array}$ \\
\hline \multicolumn{2}{|l|}{ rCDI severity at diagnosis } \\
\hline - $\quad$ Mild & $43(76.8 \%)$ \\
\hline - $\quad$ Severe/complicated & $13(23.2 \%)$ \\
\hline $\begin{array}{l}\text { Mean time from the end of anti-CD treatment for the first CDI } \\
\text { episode to the onset of rCDI diarrhea (days } \pm \text { SD) }\end{array}$ & $17.8 \pm 8.6$ \\
\hline \multicolumn{2}{|l|}{ Administered anti-CD antimicrobial treatment for rCDI } \\
\hline - Vancomycin & $34(60.7 \%)$ \\
\hline - $\quad$ PO Metronidazole & $2(3.5 \%)$ \\
\hline - $\quad$ Vancomycin and IV metronidazole & $7(12.5 \%)$ \\
\hline - $\quad$ Fidaxomicin & $10(17.8 \%)$ \\
\hline - $\quad$ Other & $3(5.3 \%)$ \\
\hline \multicolumn{2}{|l|}{ Laboratory findings at rCDI diagnosis } \\
\hline - $\quad$ Total peripheral white blood cell count $\left(10^{3}\right.$ cells $\left./ \mu \mathrm{L} \pm \mathrm{SD}\right)$ & $11,886 \pm 7912$ \\
\hline - $\quad$ Peripheral neutrophil count $\left(10^{3}\right.$ cells $\left./ \mu \mathrm{L} \pm \mathrm{SD}\right)$ & $8834 \pm 6828$ \\
\hline - $\quad$ Serum creatinine $(\mathrm{mg} / \mathrm{dL} \pm \mathrm{SD})$ & $1.5 \pm 1.2$ \\
\hline - $\quad$ Serum albumin $(\mathrm{g} / \mathrm{dL} \pm \mathrm{SD})$ & $3.3 \pm 0.6$ \\
\hline Albumin administration after rCDI diagnosis & $7(12.5 \%)$ \\
\hline Surgery for complicated rCDI & $1(1.7 \%)$ \\
\hline Necessity of intensive care & $1(1.7 \%)$ \\
\hline \multicolumn{2}{|l|}{ Outcome of rCDI } \\
\hline - $\quad$ Recovered at home & $50(89.3 \%)$ \\
\hline - $\quad$ Deceased, rCDI related & $6(10.7 \%)$ \\
\hline
\end{tabular}

\subsection{Risk Factors for rCDI}

The results of the univariate analysis are shown in Table 4. Logistic regression analysis identified exposure to cephalosporin during the 30-day follow-up and/or 3 months prior to the first CDI episode as the sole independent risk factor associated with the occurrence of rCDI (RR: 1.7; 95\% CI: 1.1-2.7, $p=0.03$ ).

Table 4. Risk factors for the occurrence of rCDI. Univariate analysis. RR: risk ratio. CI: confidence interval. SD: standard deviation. rCDI: recurrent Clostridioides difficile infection. PO: per os. IV: intravenous administration.

\begin{tabular}{|c|c|c|c|c|}
\hline Variables & $\begin{array}{c}\text { rCDI Patients } \\
(\mathrm{N}=56)(\%)\end{array}$ & $\begin{array}{l}\text { Not rCDI Patients } \\
\quad(\mathrm{N}=215)(\%)\end{array}$ & RR $(95 \%$ CI $)$ & $p$ \\
\hline Mean age $(\mathrm{y} \pm \mathrm{SD})$ & $72 \pm 15$ & $67 \pm 17$ & - & 0.08 \\
\hline Female gender & $28(50 \%)$ & $98(45.6 \%)$ & $1.2(0.7-1)$ & 0.6 \\
\hline \multicolumn{5}{|l|}{ Anti-CD treatment for the first CDI episode } \\
\hline - Vancomycin monotherapy & $38(67.8 \%)$ & $153(71.2 \%)$ & $0.9(0.5-1.5)$ & 0.6 \\
\hline - $\quad$ PO metronidazole monotherapy & $9(16.6 \%)$ & $23(10.7 \%)$ & $1.4(0.8-2.6)$ & 0.3 \\
\hline - $\quad$ Fidaxomicin (alone, in combination) & 0 & $12(5.6 \%)$ & - & 0.07 \\
\hline - $\quad$ Vancomycin plus IV metronidazole & $6(10.7 \%)$ & $30(13.9 \%)$ & $0.8(0.4-1.7)$ & 0.5 \\
\hline - $\quad$ Bezlotoxumab & $1(1.8 \%)$ & $1(0.5 \%)$ & $0.4(0.1-1.7)$ & 0.3 \\
\hline
\end{tabular}


Table 4. Cont.

\begin{tabular}{|c|c|c|c|c|}
\hline Variables & $\begin{array}{l}\text { rCDI Patients } \\
(\mathrm{N}=56)(\%)\end{array}$ & $\begin{array}{l}\text { Not rCDI Patients } \\
\quad(\mathrm{N}=215)(\%)\end{array}$ & RR $(95 \%$ CI) & $p$ \\
\hline First CDI episode severe/complicated & $22(39.3 \%)$ & $59(27.4 \%)$ & $1.5(0.9-2.4)$ & 0.08 \\
\hline Antibiotics administration during 30-day follow-up & $22(39.3 \%)$ & $74(34.4 \%)$ & $1.2(0.7-1.9)$ & 0.5 \\
\hline $\begin{array}{l}\text { Antibiotics administration during } 30 \text {-day follow-up } \\
\text { and/or } 3 \text { months prior to the first CDI episode }\end{array}$ & $52(92.8 \%)$ & $198(92.1 \%)$ & $1.1(0.4-2.7)$ & 0.8 \\
\hline - Quinolones & $17(30.3 \%)$ & $45(20.9 \%)$ & $1.5(0.9-2.4)$ & 0.1 \\
\hline - $\quad$ Penicillins & $20(35.7 \%)$ & $112(52.1 \%)$ & $0.6(0.4-1)$ & 0.03 \\
\hline - $\quad$ Carbapenems & $10(17.8 \%)$ & $38(17.7 \%)$ & $1(0.5-1.9)$ & 1 \\
\hline - $\quad$ Cephalosporins & $30(53.5 \%)$ & $74(34.4 \%)$ & $1.9(1.2-2.9)$ & 0.009 \\
\hline - $\quad$ Glycopeptides & $7(12.5 \%)$ & $25(11.6 \%)$ & $1.1(0.5-2.2)$ & 0.9 \\
\hline - $\quad$ Clindamycin & $1(1.8 \%)$ & $3(1.4 \%)$ & $1.2(0.2-6.7)$ & 0.8 \\
\hline
\end{tabular}

Finally, at the end of the follow-up period, overall crude mortality rate (either inhospital or post-discharge) was $10.7 \%$ (33/309).

\section{Discussion}

Our findings confirm that rCDI represents a relevant problem in terms of morbidity, mortality and impact on public health. Indeed, in our cohort, the incidence rate of rCDI was 72 cases per 10,000 patient-days; $21 \%(56 / 271)$ of patients with a first CDI episode developed rCDI.

There are limited data on rCDI incidence. Importantly, studies assessing rCDI incidence differ by type of cohorts included and, specially, by follow-up periods. Furthermore, most of the information on the rate of rCDI comes from studies aimed at evaluating the efficacy of anti-CDI therapies, rather than from studies specifically designed to determine it. As a consequence, rCDI incidence rates are wide-ranging [8-10], and the transferability of findings from studies assessing this rate is low.

In our cohort, the inclusion of all hospitalized patients with a first CDI episode and the rigorous methodology for assessment of recurrences allowed a trustworthy estimation of the incidence rate. We believe that reporting the incidence rate per 10,000 patient-days gives a better definition of the extent of the problem than simply reporting the percentage of rCDI patients. We are not aware of published studies that provide this information, which would make it easier to compare incidences in different cohorts.

One of the main limitations of our study pertains to the follow-up period. According to international guidelines, CDI recurrence is defined as a CDI episode that re-occurs within eight weeks after the onset of the previous CDI episode, provided symptoms from the previous episode resolve after completion of initial treatment. Our follow-up period was shorter than eight weeks after the onset of the first CDI episode; however, we preferred to consider a different time period for two main reasons: (1) the 30 days after the end of anti-CDI treatment is the period in which relapses occur most frequently [14,15]; (2) we ensured that the follow-up period was the same for all enrolled patients. In our cohort, the mean time from the end of anti-CD treatment for the first CDI episode to the new onset of diarrhea in rCDI patients was $18 \pm 9$ days. However, it is important to consider that we could have missed some cases if recurrence occurred more than 30 days after the end of CDI treatment.

Another limitation of the study is the mean age $(70 \mathrm{y})$ of the patient population. Old age was stated as a risk factor for rCDI in many countries, and the relatively high mean age of this study population may have affected results. However, it should be considered that the study population reflects consecutive CDI cases enrolled in the 15 academic or tertiary referral Italian hospitals participating in the study. 
Regarding risk factors, in our study only previous exposure to cephalosporins was independently associated with the risk of rCDI. In the literature, there are several studies evaluating the role of antibiotic exposure; antibiotics most frequently recognized as predisposing factors for $\mathrm{CDI}$ include fluoroquinolones, clindamycin and broad-spectrum penicillins and cephalosporins [16]. Regrettably, studies have evaluated different periods of exposure to antibiotics; thus, this information is frequently biased. The protective effect of penicillins that we found in our univariate analysis is difficult to explain. The majority of these patients received penicillins in the three months prior to their first CDI episode, while the percentages of $\mathrm{rCDI}$ and not-rCDI patients receiving penicillins during the 30-day follow-up decreased to $7 \%$ and $10 \%$, respectively. The univariate analyses limited to the 30-day follow-up found no significant differences between the two groups.

Our findings highlight the importance of antibiotic exposure, before the first CDI episode and after its resolution, in determining long-lasting effects on gut microbiota leading to recurrence of CDI. Physicians should sharpen their clinical judgment when choosing antibiotic therapy and strongly reinforce compliance with basic antimicrobial stewardship principles.

Finally, our study gives additional information on the outcomes of patients with primary CDI and rCDI.

Our findings on mortality are coherent with other published data; according to a meta-analysis, patients with CDI have a risk of 30-day mortality ranging between $8 \%$ and $53 \%$ [10]. Regarding the mortality rate of rCDI patients, previous studies reported rates of $9.3 \%$ [17] and $7.8 \%$ at 30 days after recurrence [18].

In conclusion, our study adds further insight into the characteristics, incidence rate, risk factors and mortality rate of rCDI. Further studies should assess the risk of antibiotic exposure by analyzing the risk associated with each antibiotic class in-depth and defining the time of exposure which should be considered at-risk. Providing the incidence rate for 10,000 days of follow-up would allow comparable and reliable data on rCDI to be obtained.

Supplementary Materials: The following are available online at https:/ / www.mdpi.com/2077-038 3/10/5/1127/s1, Table S1: List of participating centers.

Author Contributions: Conceptualization, N.P. and G.G.; methodology, M.A.C.; software, G.G., E.C. and M.A.C.; validation, N.P. and M.A.C.; formal analysis, M.A.C. and G.G.; investigation, G.G., E.C., L.A., M.B. (Matteo Bassetti), A.B., G.B., M.B. (Michele Bartoletti), P.B., G.B., R.B., G.C., A.M.D.L., S.D.B., S.F., E.F., I.G. D.R.G., E.G., F.I., F.L., G.L., A.L., L.I.L., R.L., A.E.M., M.M., M.U.M., A.M., C.M., A.O., A.P., C.R., F.F.T., M.V. and P.V.; data curation, M.B. (Michele Bartoletti), R.B., G.C., A.M.D.L., S.D.B., S.F., E.F., D.R.G., E.G., F.I., F.L., A.L., L.I.L., A.E.M., A.O., A.P., C.R. and F.F.T.; writing-original draft preparation, G.G. and E.C.; writing-review and editing, N.P. and M.A.C.; visualization, N.P.; supervision, N.P.; project administration, G.G.; funding acquisition, G.G. and N.P. All authors have read and agreed to the published version of the manuscript.

Funding: Work supported by grant by SITA GIOVANI (Young Investigators Group of the Società Italiana Terapia Antinfettiva) and by Ricerca Corrente IRCCS.

Institutional Review Board Statement: The study was conducted according to the guidelines of the Declaration of Helsinki, and approved by the Ethics Committee of National Institute for Infectious Diseases “L. Spallanzani”, IRCCS, Rome. Ethics Committee registry number: 543, 23/01/2018.

Informed Consent Statement: Informed consent was obtained from all subjects involved in the study.

Data Availability Statement: The data presented in this study are available on request from the corresponding author. The data are not publicly available due to privacy restrictions.

Acknowledgments: Lucia Adamoli, Michele Bartoletti, Alessandro Bartoloni, Gregorio Basile, Matteo Bassetti, Paolo Bonfanti, Raffaella Borromeo, Emanuela Caraffa, Maria Adriana Cataldo, Giancarlo Ceccarelli, Anna Maria De Luca, Stefano Di Bella, Sara Fossati, Erica Franceschini, Ivan Gentile, Daniele Roberto Giacobbe, Enrica Giacometti, Guido Granata Fabrizio Ingrassia, Filippo Lagi, Giambattista Lobreglio, Andrea Lombardi, Laura Isabella Lupo, Roberto Luzzati, Alberto Enrico Maraolo, Malgorzata Mikulska, Mario Umberto Mondelli, Alessandra Mularoni, Cristina Mussini, 


\begin{abstract}
Alessan-dra Oliva, Alessandro Pandolfo, Maddalena Peghin, Nicola Petrosillo, Carlotta Rogati, Antonella Santoro, Francesco Schiavone, Filippo Fabio Trapani, Mario Venditti, Pierluigi Viale.

Conflicts of Interest: Outside the submitted work, N.P. received honorary fees for conference presentations from Pfizer, MSD, Shionogi, Becton \& Dickinson and Johnson \& Johnson. In the last two years, M.V. received honorary fees for conference presentations and advisory board participations from Pfizer, MSD, Angelini, Gilead, Correvio and Menarini. Outside the submitted work, D.R.G. reports honoraria from Stepstone Pharma $\mathrm{GmbH}$ and unconditional grants from MSD Italia and Correvio Italia. Outside the submitted work, M.B. has participated in advisory boards and/or received speaker oraria from Achaogen, Angelini, Astellas, Bayer, Basilea, BioMérieux, Cidara, Gilead, Menarini, MSD, Nabriva, Paratek, Pfizer, Roche, Melinta, Shionogi, Tetraphase, VenatoRx and Vifor and has received study grants from Angelini, Basilea, Astellas, Shionogi, Cidara, Melinta, Gilead, Pfizer and MSD. A.B. received study grants from MSD, ViiV Healthcare and Nordic Pharma. The other authors declare no conflicts of interest.
\end{abstract}

\title{
References
}

1. Evans, C.T.; Safdar, N. Current Trends in the Epidemiology and Outcomes of Clostridium difficile Infection. Clin. Infect. Dis. 2015, 60 (Suppl. 2), S66-S71. [CrossRef] [PubMed]

2. Magill, S.S.; Edwards, J.R.; Bamberg, W.; Beldavs, Z.G.; Dumyati, G.; Kainer, M.A.; Lynfield, R.; Maloney, M.; McAllister-Hollod, L.; Nadle, J.; et al. Multistate point-prevalence survey of health care-associated infections. N. Engl. J. Med. 2014, 370, 1198-1208. [CrossRef] [PubMed]

3. European Centre for Disease Prevention and Control. Healthcare-Associated Infections: Clostridium Difficile Infections; Annual Epidemiological Report for 2016; ECDC: Stockholm, Sweden, 2018.

4. McFarland, L.V. Renewed interest in a difficult disease: Clostridium difficile infections-epidemiology and current treatment strategies. Curr. Opin. Gastroenterol. 2009, 25, 24-35. [CrossRef] [PubMed]

5. McFarland, L.V.; Elmer, G.W.; Surawicz, C.M. Breaking the cycle: Treatment strategies for 163 cases of recurrent Clostridium difficile disease. Am. J. Gastroenterol. 2002, 97, 1769-1775. [CrossRef] [PubMed]

6. Davies, K.A.; Ashwin, H.; Longshaw, C.M.; Burns, D.A.; Davis, G.L.; Wilcox, M.H.; EUCLID Study Group. Diversity of Clostridium difficile PCR ribotypes in Europe: Results from the European, multicentre, prospective, biannual, point-prevalence study of Clostridium difficile infection in hospitalized patients with diarrhoea (EUCLID), 2012 and 2013. Euro Surveill. 2016, 21. [CrossRef] [PubMed]

7. Guillemin, I.; Marrel, A.; Lambert, J.; Beriot-Mathiot, A.; Doucet, C.; Kazoglou, O.; Luxemburger, C.; Reygrobellet, C.; Arnould, B. Patients' experience and perception of hospital-treated Clostridium difficile infections: A qualitative study. Patient 2014, 7, 97-105. [CrossRef] [PubMed]

8. Pepin, J.; Alary, M.E.; Valiquette, L.; Raiche, E.; Ruel, J.; Fulop, K.; Godin, D.; Bourassa, C. Increasing risk of relapse after treatment of Clostridium difficile colitis in Quebec, Canada. Clin. Infect. Dis. 2005, 40, 1591-1597. [CrossRef] [PubMed]

9. Sheitoyan-Pesant, C.; Abou Chakra, C.N.; Pepin, J.; Marcil-Heguy, A.; Nault, V.; Valiquette, L. Clinical and healthcare burden of multiple recurrences of Clostridium difficile infection. Clin. Infect. Dis. 2016, 62, 574-580. [CrossRef] [PubMed]

10. Abou Chakra, C.N.; Pépin, J.; Sirard, S.; Valiquette, L. Risk factors for recurrence, complications and mortality in Clostridium difficile infection: A systematic review. PLoS ONE 2014, 9, e98400. [CrossRef] [PubMed]

11. Brandt, L.J.; Aroniadis, O.C.; Mellow, M.; Kanatzar, A.; Kelly, C.; Park, T.; Stollman, N.; Rohlke, F.; Surawicz, C. Long-term follow-up of colonoscopic fecal microbiota transplant for recurrent Clostridium difficile infection. Am. J. Gastroenterol. 2012, 107, 1079-1087. [CrossRef] [PubMed]

12. Rodriguez, C.; Taminiau, B.; Van Broeck, J.; Delmée, M.; Daube, G. Clostridium difficile infection and intestinal microbiota interactions. Microb. Pathog. 2015, 89, 201-209. [CrossRef] [PubMed]

13. Debast, S.B.; Bauer, M.P.; Kuijper, E.J. European Society of Clinical Microbiology and Infectious Diseases: Update of the treatment guidance document for Clostridium difficile infection. European Society of Clinical Microbiology and Infectious Diseases. Clin. Microbiol. Infect. 2014, 20 (Suppl. 2), 1-26. [CrossRef] [PubMed]

14. Kelly, C.P. Can we identify patients at high risk of recurrent Clostridium difficile infection? Clin. Microbiol. Infect. 2012, 18, 21-27. [CrossRef] [PubMed]

15. Lowy, I.; Molrine, D.C.; Leav, B.A.; Blair, B.M.; Baxter, R.; Gerding, D.N.; Nichol, G.; Thomas, W.D., Jr.; Leney, M.; Sloan, S.; et al. Treatment with monoclonal antibodies against Clostridium difficile toxins. N. Engl. J. Med. 2010, 362, 197-205. [CrossRef] [PubMed]

16. Deshpande, A.; Pasupuleti, V.; Thota, P.; Pant, C.; Rolston, D.D.; Hernandez, A.V.; Donskey, C.J.; Fraser, T.G. Risk factors for recurrent Clostridium difficile infection: A systematic review and meta-analysis. Infect. Control Hosp. Epidemiol. 2015, 36, 452-460. [CrossRef] [PubMed] 
17. Pépin, J.; Routhier, S.; Gagnon, S.; Brazeau, I. Management and Outcomes of a First Recurrence of Clostridium difficile-Associated Disease in Quebec, Canada. Clin. Infect. Dis. 2006, 42, 758-764. [CrossRef] [PubMed]

18. Olsen, M.A.; Yan, Y.; Reske, K.A.; Zilberberg, M.D.; Dubberke, E.R. Recurrent Clostridium difficile infection is associated with increased mortality. Clin. Microbiol. Infect. 2015, 21, 164-170. [CrossRef] [PubMed] 